\title{
Two-extremum electrostatic potential of metal-lattice plasma and the work function of an electron
}

\author{
S.A. Surma, J. BronA*, A. Ciszewski \\ Institute of Experimental Physics, University of Wroclaw, Plac Maxa Borna 9, 50-204 Wroclaw, Poland
}

\begin{abstract}
Metal-lattice plasma is treated as a neutral two-component two-phase system of 2D surface and 3D bulk. Free electron density and bulk chemical potential are used as intensive parameters of the system with the phase boundary position determined in the crystalline lattice. A semiempirical expression for the electron screened electrostatic potential is constructed using the lattice-plasma polarization concept. It comprises an image term and three repulsion/attraction terms of second and fourth orders. The novel curve has two extremes and agrees with certain theoretical forms of potential. A practical formula for the electron work function of metals and a simplified schema of electronic structure at the metal/vacuum interface are proposed. This yields $10.44 \mathrm{eV}$ for the Fermi energy of free electron gas; $-5.817 \mathrm{eV}$ for the Fermi energy level; $4.509 \mathrm{eV}$ for the average work function of bcc tungsten. Selected data are also given for fcc $\mathrm{Cu}$ and hcp Re. For harmonic frequencies $\sim 10 \mathrm{E} 16$ per s of the self-excited metal-lattice plasma, energy gaps of 14.54 and $8.02 \mathrm{eV}$ are found, which correspond to the bulk and surface plasmons, respectively. Further extension of this thermodynamics and metal-lattice theory based approach may contribute to a better understanding of theoretical models which are employed in chemical physics, catalysis and materials science of nanostructures.
\end{abstract}

Keywords: under-coordinated system; surface and interface; screened potential; metal-lattice plasma density; work function; electron affinity

(C) Wroclaw University of Technology.

\section{Introduction}

In metals theory based on valence electrons, different pseudopotential approximations are employed to compensate the ion core Coulomb potential [1-6]. One of such approaches together with density-functional theory [7] based approximations and perturbation methods, for treating the electron interactions in different states of matter and elements, is the pseudopotential plane wave method, apart from other or mixed methods. For solutions of bio-molecular structures, Tavares and Prausnitz have performed comparative calculations of phase diagrams [8] by employing the Barker-Henderson second-order perturbation theory. There are also phenomenological approaches to the electron work function (WF) which is one of the most important characteristics of materials. In order to ensure a wide area of applicability to metals and alloys, such

\footnotetext{
*E-mail: jacek.brona@ifd.uni.wroc.pl
}

approaches use the classical electrostatic image or the unscreened Coulomb potential [9-15].

Brodie, not employing an ab initio method, has calculated the WF of the polycrystalline surface in terms of the conducting-sphere radius and the Fermi energy, obtained a good agreement with experimental data for several fcc and bcc metals [10], and has recently generalized his model of WF [11]. Using Brodie's concept as the starting point, Halas and Durakiewicz (H-D) have combined the polarization length of metal-lattice plasma (MLP) with the Fermi energy to calculate the polycrystalline WF of sixty elements [12]. Their new model [13], using the free-electron gas and classical electrodynamics approach, leads to a good agreement with WF experimental data concerning metals, rare earth elements as well as alloys and yields the most reliable data despite its phenomenological character [9].

Such results of the H-D approach were motivation for the present attempt at the electron screened 
potential and WF problems. For constructing our pseudopotential $\Psi_{\mathrm{ES}}(\mathrm{x})$ and a simple formula of average WF proposed, the plasma polarization concept has been developed and two intensive parameters of the 2D plasma included. Since the 3D metal inside exhibits isotropic electronic properties, while its 2D surface skin of 2 or 3 atomic layers and the WF display clearly anisotropic ones, we treat a crystal of metal (Me) as a thermodynamic two-phase two-component system of free electrons and lattice ions. The characteristic length $\mathrm{d}_{\mathrm{s}}(\mathrm{hkl})$ which determines the phase boundary position between the Me surface and the bulk, was determined [16] in terms of the crystalline (atomic) lattice. The novel potential $\Psi_{\mathrm{ES}}$ with its two extremes has the form similar to theoretical pseudopotentials, such as the Tavares-Prausnitz double minimum potential $[8,17]$.

\section{Base for the calculations}

\subsection{Formalism}

Formulae are given in the system of units CGS ESU; the unitary vacuum permittivity $\varepsilon_{0}$ is omitted. The units $1 \AA\left(=1 \times 10^{-10} \mathrm{~m}\right)$, $1 \mathrm{eV}\left(\approx 1.602 \times 10^{-19} \mathrm{~J}\right)$ and $1 \mathrm{D}$ (debye $\approx$ $\left.3.3356 \times 10^{-30} \mathrm{C} \cdot \mathrm{m}\right)$ are also used in this paper. Physical and chemical potentials and potential energies refer to an electron with zero chosen at infinity in the vacuum. Calculations are made for tungsten with its intensive parameter of MLP the chemical potential found by employing the linear regression method for the hard-sphere system [16].

The metal bulk is defined in the first nearest neighbour approximation employing the broken bonds model [18]. The interior of the crystal has the zero total number of atoms with broken u-bonds, i.e. where the surface dipole layer (double layer) of electrostatic charge (DL) is absent, which occurs for $\mathrm{x} \leqslant-\mathrm{d}_{\mathrm{s}}(\mathrm{hkl})$. This is illustrated in Fig. 4 of Appendix. The ideal half-crystal's surface of metal is unbounded in its two tangential dimensions, but is bounded in the normal direction by two infinite planes with the characteristic length $d_{s}$ in between. For the definition of $\mathrm{d}_{\mathrm{s}}(\mathrm{hkl})$ (Appendix). It should be noted that the phase boundary position $d_{s}(h k l)$ is independent of Miller indices parity.

Metal's average surface (MS) skin was defined for a conventional polycrystal [16]. For bcc metals, the MS of a metal can best be illustrated with the (111) plane as shown in Fig. 4. The term 'physical surface' refers to the conventional surface which is composed of nondegenerate or thermalized electrons - the "electron cloud" of Smoluchowski's [19]. The origin of the coordinate system is placed on the geometrical surface, or the first lattice plane with $x>0$ on the side of the vacuum. The $\mathrm{x}^{\prime}$ coordinate (equal to $\mathrm{x}-\mathrm{x}_{0}$ ) are the distances measured from half the primary (external) electron-image plane position. The origin shift $\mathrm{x}_{0}$ is taken equal to polarization length of MLP. To compute this length we utilize the DebyeHueckel screening radius L of classical plasma physics [20], which corresponds to the screening length of Thomas-Fermi approximation. Both these characteristic lengths, $d_{s}$ and $L$, are attributed to the infinite two-dimensional, anisothermal metallattice plasma [21-23], which can locally be thermalized and polarized, as its two linear dimensions.

\subsection{Polarized metal-lattice plasma}

An electrically neutral semi-infinite crystal of Me is treated in the following as a two-phase thermodynamic system of the isotropic bonded bulk $\mathrm{Me}(\mathrm{BM})$ phase with delocalized valence electrons and the Me surface phase composed of 2 or 3 layers of atoms with directional bonds within the surface skin. The high-density low temperature MLP constitutes a two-component system of electrons and lattice ion cores (i.e. the conventional positive ions) in the above two phases. Such a neutral twocomponent two-phase system of free electrons and ion cores has two degrees of freedom. In view of the Gibbs phase rule, the two degrees of freedom of MLP permit the free electron number density $n$ and the bulk chemical potential $\mu$ to be treated as two intensive parameters which are a function of position and independent of the system's size.

Plasma of lattice ions and free electrons is anisothermal in the BM phase, where the FermiDirac distribution is valid. It may, however, be thermalized within some locally adiabatic regions 
of MS phase. In the local thermal equilibrium $\left(\mathrm{T}_{\mathrm{i}} \approx \mathrm{T}_{\mathrm{e}}\right.$ ), polarization of the surface phase occurs and the dipole barrier exists. The resulting facedependent variation in intrinsic field strength at the surface is considerable and accounts for the work function anisotropy. The MS phase is treated as a self-excited resonance system which is associated with the intrinsic fields. To characterize the locally polarized plasma, essential are its local electron density parameter $\mathrm{n}_{\mathrm{x}}$ (associated with the linear dimensions of the MLP space) and the local Fermi energy of the metal.

For both sides of the Me/Vacuum interface, we assume the MLP to have the dielectric constant or pseudo-permittivity in the range $0<\varepsilon \leqslant 1$. This corresponds to typical MLP densities of 0.2 to 5.2 $\times 10^{23} \mathrm{~cm}^{-3}$. Then, like the case of gas discharge plasmas, enhancement of local electric field occurs rather than the dielectric-type reduction [24]. The above accounts for the normal translational kinetic energy, $\mathrm{mv}_{\mathrm{x}}^{2} / 2$, of electrons and also for the energy of simple harmonic oscillations of MLP owing to possible lateral small displacements of surface ions and electrons occurring on metastable sites in yzplane. The traditional picture below needs the assumption of treating the MLP as a quasi-elastic medium.

Let a local MLP density in a stable thermal equilibrium be equal to $\mathrm{n}_{\mathrm{x}}$ and the changes in density very small around the equilibrium position. Then an electron may behave as a natural, small $\mathrm{a}_{1}-$ amplitude oscillator. The lateral electric restoring force $\mathrm{qF}_{1}$ of $-4 \pi \mathrm{n}_{\mathrm{x}} \mathrm{e}^{2} \mathrm{a}_{1}$ may appear at every gravity $\mathrm{x}$-centre of oscillating charges, and the energy of harmonic oscillation is:

$$
E_{t}=\frac{m \omega_{p}^{2} a_{l}^{2}}{2}
$$

with the function $\omega_{\mathrm{p}}\left(\mathrm{n}_{\mathrm{x}}\right)$ being the plasma resonance angular frequency given by:

$$
\omega_{p}^{2}=\frac{4 \pi n_{x} e^{2}}{m}
$$

where $\mathrm{m}$ is the electron mass and $\mathrm{e}$ is the elementary charge. Then the energy $\mathrm{E}_{\mathrm{t}}$ is expressed as:

$$
2 \pi n_{x} e^{2} a_{l}^{2}
$$

Note, the electron mass does not enter the final formula of harmonic oscillation energy.

In such a case, the total energy per electron of the 2D plasma in the Me/Vacuum interface can be expressed in the Cartesian coordinate system as:

$$
E=\bar{U}(x)+\frac{m v_{x}^{2}}{2}+\pi n_{x} e^{2} a_{l}^{2}
$$

The terms on the right-hand side of equation represent average values of the potential energy, the translational kinetic energy, and the energy $\tilde{\mathrm{E}}_{\mathrm{t}}$ of harmonic small oscillation, respectively.

\section{The MLP electron density pa- rameter}

The H-D spontaneous polarization length [12] may be identified with the linear dimension of the 2D MLP or the Thomas-Fermi screening length. In contrast to the H-D procedure, to calculate the polarization length we use the local electron density $\mathrm{n}_{\mathrm{s}}$ that is related to the MS phase rather than the free electron average density $\tilde{n}$. We assume that the MLP linear dimension is concerning the case of locally thermalized plasma $\left(\mathrm{T}_{\mathrm{e}} \approx \mathrm{T}_{\mathrm{i}}\right)$. Consequently, for the Fermi energy, we will put the local Fermi energy $E_{k x}$ of the MS phase into the relation for the Debye-Hueckel screening length L of classical plasma:

$$
L=\left[\frac{k T_{i}}{4 \pi n e^{2}\left(1+\frac{T_{i}}{T_{e}}\right)}\right]^{1 / 2}=\left[\frac{E_{k x}}{8 \pi n e^{2}}\right]^{1 / 2}
$$

Density $n$ will be replaced with the local density $\mathrm{n}_{\mathrm{s}}$ which is (hkl)-dependent and defined in terms of the face dependent distorted radius $\mathrm{R}_{\mathrm{d}}$ of an MS "polarized" atom. We assume that the surface ion becomes practically screened above a distance of twice the Thomas-Fermi screening radius $1 / \mathrm{k}_{\mathrm{s}}$, or from about $2 \mathrm{~L}$ to $\mathrm{x}_{\mathrm{s}}$.

The local Fermi kinetic energy $\mathrm{E}_{\mathrm{F}, \mathrm{s}}$ of an electron, like other respective Fermi energies, is simply obtained from the classical Sommerfeld relation for the kinetic energy of free electron gas:

$$
E_{F, s}=\frac{1}{2} \hbar^{2} m^{-1}\left(3 \pi^{2} n_{s}\right)^{2 / 3}
$$


In order to calculate the density $\mathrm{n}_{\mathrm{s}}$, the MLP linear dimension $d_{s}$ will be used. First, it is proper to take into account the size of surface atoms. Using a conventional diameter $\left(2 R_{a}-2 l_{h}\right)$ of an MS positive "ion" we drive at a relation for a surface polarized atom:

$$
7 R_{d(M S)}=d_{s(M S)}-R_{a}+l_{h(M S)} \approx R_{a}+1 / 2 l_{(M S)}
$$

where $l_{h}$ is the dipole half-length of DL, and $R_{a}$ is the atomic (metallic) radius. The (hkl)-dependent dipole length, $\mathrm{l}_{(\mathrm{hkl})}$, of the DL moment $\mathrm{p}$ is a semiempirical factor associated with the MS:

$$
l_{M S}=\frac{B d_{s(M S)}}{2 \pi e^{2}}
$$

where $\mathrm{B}$ is a constant numerically equal to the slope of the linear regression [16]; henceforth, we omit subscript MS. The radius $R_{d}$ is related to the average MS electron density by:

$$
\frac{1}{n_{s}}=S_{0} R_{d}
$$

where $S_{0}$ is the area of a $2 D$ primitive unit cell of the MS (Appendix). Let the product $\mathrm{S}_{0} \mathrm{l}$ be associated with the unit volume of the locally polarized 2D plasma, and denoted $\mathrm{v}_{0}$. The sphere radius $\mathrm{r}_{0}$ corresponding to the $\mathrm{v}_{0}$ approximately equals $3 / 2$ a.u. (bohr) and is comparable with typical Debye lengths of 0.5 to $1.0 \AA$. The related unit density, $\mathrm{v}_{0}^{-1}$, is denoted $\mathrm{n}_{0}$.

For an electrically neutral metal, the MLP electrons are leaking out of the surface into the vacuum to condense and create the physical surface. The ideal physical surface is located about the plane of equilibrium between attraction and repulsion forces. It is distant from the geometrical surface by:

$$
x_{s}=R_{d}+x_{0}
$$

True position of the physical surface is diffuse by $\pm \mathrm{x}_{0}$ (Fig. 1). Distorted spherical symmetry of MS-phase atoms (and Wigner-Seitz cells at the surface) is reflected by the radius $R_{d}$. In the hardsphere approximation, this surface phase parameter determines the equilibrium position $\left(\mathrm{x}_{\mathrm{s}} \pm \mathrm{x}_{0}\right)$ of the physical surface and can be treated as invariant with respect to the attraction potentials [25, 26].

Intensive parameter the average density of free electrons, $\tilde{n}$, is defined as the inverse of the volume per atom of Me, reduced by the conventional volume of an MLP ion itself, i.e. using the van der Waals-type correction:

$$
\frac{1}{\tilde{n}}=\Omega-\frac{4}{3}\left(R_{a}-l_{h}\right)^{3}
$$

where $\Omega$ is the volume per atom of the crystal.

Density $\mathrm{n}^{+}$is associated with some surface ions of the charge +1 . It is the primary positive component of MLP density which, in a series with multiply charged ions, is introduced to satisfy the charge neutrality condition:

$$
\frac{1}{n^{+}}=S_{0} d_{s}
$$

The electric neutrality condition for the total concentration $n$ of electrons can be expressed as:

$$
n=\sum z_{i} n_{i}^{+}
$$

where $\mathrm{i}=\mathrm{z}_{\mathrm{i}}=1,2,3, \ldots, \mathrm{m}$ and the first term $\mathrm{n}^{+}$is assumed equal to the concentration of atoms with the average deficiency of one electron per atom, the second term is related to the ones with a double electron deficiency etc. up to a limiting deficiency of $z_{m}$ electrons per atom of MS. A local, or notional, deviation of the $\mathrm{n}(\mathrm{x})$ density from the neutrality condition is manifested as plasma polarization. Characteristic lengths and magnitudes of parameters of the key quantities, together with some magnitudes of interest for surface science, are given in Tables 1 and 2 . The respective potentials, Fermi energies obtained from equation 6 , and other data are collected in Tables 3 and 4.

\section{Electron work function}

The work function of an electron of crystal, which is also called the effective WF and usually denoted by the letter $\varphi$, is a fundamental physicochemical characteristic of metal and semiconductor surfaces and interfaces. Different approaches to the 
Table 1. Magnitudes of characteristic lengths and quantities.

\begin{tabular}{|c|c|c|}
\hline Quantity & Tungsten W [unit] & Remarks \\
\hline MLP pseudo-permittivity $\varepsilon$ & 0.303 & Cu: 0.413 , Re: 0.311 \\
\hline MS primitive unit cell area $S_{0}$ & $1.494 \mathrm{a}^{2}$ & \\
\hline MLP unit volume $\mathrm{v}_{0}$ & $1.92 \AA^{3}$ & \\
\hline effective dipole length 1 & $0.1283 \AA$ & $\mathrm{Cu}: 0.13, \mathrm{Re}: 0.10$ \\
\hline MLP polarization length $\mathrm{L}$ & 0.529 & $\mathrm{Cu}: 0.503$, Re: 0.553 \\
\hline Brodie critical distance $^{\mathrm{a}} \mathrm{d}$ & 0.605 & {$[10]$} \\
\hline distorted radius $\mathrm{R}_{\mathrm{d}}$ & 1.431 & \\
\hline phase boundary position $d_{s}$ & 2.737 & $\mathrm{Cu}: 2.685, \mathrm{Re}: 2.95$ \\
\hline outside/vacuum site $\mathrm{x}_{\mathrm{c}}$ & 3.266 & \\
\hline physical surface position $\mathrm{x}_{\mathrm{s}}$ & $1.96 \pm 0.529$ & $\mathrm{r}_{\mathrm{s}}=3.7 \pm 1.0$ \\
\hline pseudopotential's maximum $\mathrm{x}_{\mathrm{m}}$ & 1.90 & \\
\hline pseudopotential's minimum $\mathrm{x}_{\mathrm{n}}$ & 3.58 & \\
\hline inflexion points $A_{1}$ and $A_{0}$ & $2.2 ; 5.3$ & \\
\hline metallic radius $R_{a}$ & 1.37 & $\mathrm{Cu}: 1.28, \mathrm{Re}: 1.37$ [52] \\
\hline lattice parameter a & 3.165 & $\mathrm{Cu}: 3.615$, Re: 2.761 accepted \\
\hline
\end{tabular}

${ }^{a}$ Identified [10] with the Heisenberg uncertainty distance $\delta x$ related to the $\left(2 \mathrm{E}_{\mathrm{F}} \mathrm{m}_{\mathrm{eff}}\right)^{\frac{1}{2}}$ momentum change to zero just after an electron's emission.

Table 2. Magnitudes of parameters and quantities for the electron screened potential.

\begin{tabular}{|c|c|c|}
\hline Quantity & Tungsten W [unit] & Remarks \\
\hline MS positive charge density $\mathrm{n}^{+}$ & $0.244 \times 10^{23} \mathrm{~cm}^{-3}$ & \\
\hline MS electron density $n_{s}$ & $0.467 \times 10^{23}$ & \\
\hline free electron ${ }^{\mathrm{a}}$ average density $\tilde{\mathrm{n}}$ & $1.533 \times 10^{23}$ & $\mathrm{Cu}: 2.7 \times 10^{23}, \mathrm{Re}: 2.0 \times 10^{23}$ \\
\hline local electron ${ }^{b}$ density $n_{(100)}$ & $4.010 \times 10^{23}$ & \\
\hline MS unit density $\mathrm{n}_{0}$ & $5.207 \times 10^{23}$ & \\
\hline limiting density $\mathrm{n}^{*}\left(\mathrm{R}_{\mathrm{d}}\right)$ & $21.2 \times 10^{23}$ & \\
\hline limiting density $n\left(r_{s}=1\right)$ & $16 \times 10^{23}$ & [7] formula (86) \\
\hline theoretical electron density $n$ & $3.8 \times 10^{23}$ & $\mathrm{r}_{\mathrm{s}}=1.62[27]$ \\
\hline dipole polarizability $\alpha_{0}$ & $1.53 \times 10^{-25} \mathrm{~cm}^{3}$ & \\
\hline electron polarizability & $3.52 \times 10^{-25}$ & estimated \\
\hline $\mathrm{W}(110)$ atom polarizability $\alpha_{110}$ & $5.4 \times 10^{-25}$ & {$[53]$} \\
\hline MLP local moment $\mathrm{p}_{0}$ & $0.616 \mathrm{D}$ & \\
\hline DL moment magnitude $\mathrm{p}$ & 0.187 & \\
\hline $\mathrm{W}(110)$ adatom momen ${ }^{\mathrm{c}} \mathrm{p}$ & 0.2 & {$[54]$} \\
\hline intrinsic barrier field F & $-3.359 \mathrm{~V}^{-1}$ & \\
\hline lateral field component $F_{1}$ & 1.085 & \\
\hline normal field component $\mathrm{D}_{\mathrm{x}}$ & 3.663 & $\mathrm{Cu}: 6.78, \mathrm{Re}: 3.3$ \\
\hline virtual field component $F_{\mathrm{v}}$ & 12.09 & $\mathrm{Cu}: 16.4$, Re: 10.6 \\
\hline
\end{tabular}

${ }^{a}$ Value almost equal to that for the most packed plane W(110).

${ }^{\mathrm{b}}$ Same value was used to model polarizability in the Hohenberg-Kohn theory of electron gas [7].

${ }^{\mathrm{c}}$ Measured for W/W(110) adatom surface-diffusion by FIM. (1D $\equiv 1$ debye $\left.=10^{-18} \mathrm{ESU}\right)$. 
work function problem were discussed in [27]. The WF has been defined in the literature in different ways [27-31], principally the following two ones.

- The WF of an electron of a metal was defined $[32,33]$ as the difference in energy between a lattice with an equal number of ions and electrons, and the lattice with the same number of ions, but with one electron removed from the highest energy state of the neutral metal. It can be expressed by the formula:

$$
W F=e \Delta \Phi_{(h k l)}-\mu
$$

where $\mu$ is the bulk chemical potential, and $\mathrm{e} \Delta \Phi_{(\mathrm{hkl})}$ is the potential change responsible for the DL contribution to the WF. The $\mathrm{e} \Delta \Phi$ is face dependent, while the internal intensive parameter $\mu$ is homogeneous, which was proved by Smoluchowski [19]. Magnitudes of the chemical potential $\mu$ were found for several $\mathrm{d}$ transition metals based on a great number of existing experimental WF values [16]; there was also the effective WF change $\Delta \varphi_{(\mathrm{hkl})}$ determined using the Helmholtz 2D-gas dipole formula.

- A thermodynamics based approach was that by Herring and Nichols [34]. The WF of an electron of a metal crystal was formulated as the difference between the electrochemical potential of metal electrons, taken per electron, and the electrostatic potential energy of an electron at a point in vacuum just outside the surface:

$$
W F=e \Psi_{c}-\tilde{\mu}
$$

where $\tilde{\mu}$ is the electrochemical potential, and $\Psi_{\mathrm{c}}(\mathrm{x})$ is the outer electrostatic (Volta) potential $\leqslant 0$. The electrochemical potential $\tilde{\mu}$ is defined using the notion of inner potential $\Psi$ of a phase in the electrochemical system [35]. Consequently:

$$
\tilde{\mu}=\mu+\Phi_{v}=\mu^{0}+\Phi_{s}
$$

where $\mu^{0}$ is the standard chemical potential of an electron of metal at constant temperature; it is computed from the condition 16 .
On the other hand:

$$
W F=-e \chi_{s}-\mu^{0}
$$

where $\chi_{s}$ is the effective surface potential change which is face dependent. For this two-phase system, the $\Delta \Phi$ represents the difference in inner potential between the phases MS and BM, $\Phi_{\mathrm{v}}-\Phi_{\mathrm{s}}$, across the phase boundary situated at $-\mathrm{d}_{\mathrm{s}}$. Value of $-\mathrm{e} \chi_{\mathrm{s}}$ equals double the WF change $\Delta \varphi$.

The inner potential $\Phi_{\mathrm{s}}$ is also face dependent; it is defined in terms of electrochemistry as the sum of the average outer potential $\Psi_{\mathrm{c}}$ and the effective surface potential change $\chi_{s}$. The average bulk potential $\Phi_{\mathrm{v}}$ is computed from the expression $-4 \pi \mathrm{p}_{0} / \Delta \mathrm{S}$, where $\Delta S$ is replaced with $S_{0}$ and the magnitude of the MLP local dipole moment $\mathrm{p}_{0}$ taken equal to the product el.

For a neutral MS (polycrystalline) metal in the absence of applied field, the nearsurface level $\Psi_{\mathrm{c}}$ of the outer potential is being calculated by realizing purely electrostatic virtual works $\delta \mathrm{W}$ associated with the infinitesimal drops in potential energy, $-\delta \mathrm{e} \Psi_{\mathrm{x}}$ each. These works are executed by infinitesimally slow bringing a massless test particle of the elementary charge $\mathrm{e}$, in a quasi-static reversible process, from infinity in the vacuum under action of the long range image force down to the position $\mathrm{x}_{\mathrm{c}}$ outside the Me surface. In such a reversible process being a dense set of consecutive equilibrium states, the closest surface atom still retains its original screening; then the dipole repulsion together with the electron exchange/correlation (XC) effects may be neglected. Thus, the image force is applicable safely down to the point $\mathrm{x}_{\mathrm{c}}$ equal to or higher than $d_{s}+x_{0}$. The outer potential level $\Psi\left(\mathrm{x}_{\mathrm{c}}\right)$ is then calculated by integration:

$$
\Psi_{c}=e^{-1} W_{x \leftarrow \infty}=e^{-1} \int_{\infty}^{x_{c}} \frac{-e^{2}}{4\left(x-x_{0}\right)^{2}} d x=-\Delta \Psi_{\infty, x}
$$


where $\Psi_{\mathrm{x}=\infty}=0$. This gives $\Psi_{\mathrm{c}}=-\mathrm{e} / 4 \mathrm{~d}_{\mathrm{s}}$. For $\mathrm{x}<\mathrm{x}_{\mathrm{c}}$, however, the $\mathrm{XC}$ interactions cannot be ignored.

The theoretical work function $\left(\mathrm{WF}_{\infty}\right)$ is commonly identified with the energy needed to remove an electron of a metal from the Fermi level into the vacuum to a point at infinity, i.e. taking a zero outer electrostatic potential $\Psi_{\mathrm{c}}(\mathrm{x})$. The WF values experimentally obtained are smaller.

- An approximate formula can be obtained for the average WF via the following total energy consideration. In virtue of the energy conservation law, upon removing an electron just outside the surface, the average change in total energy of the system of interest is zero:

$$
\Delta E=\Delta \bar{U}+\Delta \bar{E}_{k}=0
$$

where the energy $\mathrm{E}$ is expressed by equation 4 and the differences are taken between the relevant energy levels. Assigning the plus sign to the quantities related to the transient activation, such as the mean energy quantum $\tilde{\mathrm{h}} \boldsymbol{v}$ and the MLP excitation energy $\tilde{\mathrm{E}}_{1}$, while the minus sign to formal energy losses for the thermodynamic system, we have:

$$
0=-\tilde{\mu}-E_{F}(\tilde{n})+\tilde{h v}+\tilde{E}_{t}
$$

and finally, taking $\tilde{\mathrm{hv}}=\mathrm{WF}$, we get:

$$
W F=\tilde{\mu}+E_{F}(\tilde{n})-\tilde{E}_{t}
$$

Such an estimated average electron WF is in nice accord with the values of effective WF from the literature. The minimum work function $\mathrm{WF}_{\mathrm{m}}$ is then obtained as the sum of the least possible energy loss equal to $-\mu^{0}$ and the average energy gain $\tilde{\mathrm{E}}_{\mathrm{t}}$, i.e. as $\tilde{\mathrm{E}}_{\mathrm{t}}-\mu^{0}$.

An average electron affinity, $A_{a t}$, with respect to a neutral surface atom of $1 / 2 \mathrm{Me}_{2}$ can be estimated in the same way. An incoming test electron is to lose an energy of $A_{a t}$ in favour of the system. Relevant equations are:

$$
0=\mu^{0}+E_{F, s}+\tilde{E}_{t}-A_{a t}
$$

$$
A_{a t}=\mu^{0}+E_{F, s}+\tilde{E}_{t}=V_{1}-V_{2}
$$

where $V_{1}-V_{2}=\left(A_{0}+A_{1}\right) / 2-A_{2}$. The $A_{0}$ is the small-oscillation ground level corresponding to a metastable surface state. The $A_{0}$ and $A_{1}$ levels, that pass through the two inflection points shown in the figures, reflect virtual bound states or the surface states receptive to electron tunneling during emission. The difference $\Delta \mathrm{A}$, interpreted as the resonance energy, is almost equal to the value of $\tilde{\mathrm{E}}_{\mathrm{t}}$ (Tables 3 and 4).

\section{The electron screened electro- static potential $\Psi_{\mathrm{ES}}$}

First, we should have estimated the dielectric constant $\varepsilon$ of the MS phase within the Me/Vacuum interface. The vacuum dielectric constant equal to unity is usually ascribed to metals. The force $f_{x}$, normal to the surface and acting on an electron between two infinite planes at a short distance slightly higher than $R_{d}$ from the geometrical plane, is expressed by the product $-\mathrm{eD}_{\mathrm{x}}$. The normal component $D_{x}$ of the effective field displacement vector $\mathbf{D}$ is ascribed to the local MLP polarization, and represents the effective field $4 \pi \bar{\sigma}$ of the surface layer. The corresponding average surface potential level $\chi_{s}$ is equal to $-4 \pi \bar{\sigma} l$. The field strength component $\mathrm{D}_{\mathrm{x}} / \varepsilon$ represents the virtual intrinsic field $\mathrm{F}_{\mathrm{V}}$ of the magnitude $4 \pi \sigma$, and depicts the force acting along the polarized plasma medium. The charge distribution is discrete, and respective field components vary over the DL region, like those of an electric dipole's field.

The pseudo-permittivity $\varepsilon$ can be replaced with the factor $\theta$ being a real number such that $0<$ $\theta \leqslant 1$. The continuous distribution of the surface charge density $\bar{\sigma}$ at the physical surface is approximated by the average density $\Delta q / \Delta S$. It can be expressed as the ratio $\vartheta \mathrm{e} / \mathrm{S}_{0}$, where $0<\vartheta \leqslant 1$. Then:

$$
D_{x}=\frac{4 \pi \theta e}{S_{0}}
$$

where $\theta$ is a surface charge redistribution factor defined as the ratio $\left(\vartheta^{-}-\vartheta^{+}\right) / \vartheta_{\max }$ with the $\vartheta_{\max }$ equal to unity. The $\theta$ factor has been calculated using equations (3), (7) and (8) of paper [16]. The 
Table 3. Values of potentials of an electron.

\begin{tabular}{lcc}
\hline \multicolumn{1}{c}{ Quantity } & Tungsten $\mathrm{W}$ [unit] & Remarks \\
\hline \hline effective potential $\mathrm{V}_{0}(\mathrm{~min})$ & $-0.53 \mathrm{~V}$ & \\
pseudopotential $\Psi_{\mathrm{ES}}(\mathrm{min})$ & -0.554 & \\
surface potential change $\chi_{\mathrm{s}}$ & -0.470 & \\
outer potential level $\Psi_{\mathrm{c}}$ & -1.315 & \\
MS inner potential $\Phi_{\mathrm{s}}$ & -1.785 & \\
bulk average potential $\Phi_{\mathrm{v}}$ & -1.551 & \\
MS chemical potential $\mu^{0}$ & $-4.032 \mathrm{eV}$ & \\
bulk chemical $^{\mathrm{a}}$ potential $\mu$ & -4.267 & {$[16]$} \\
Fermi level $\tilde{\mu}$ & -5.817 & $\mathrm{Cu}: 5.96$, Re: 6.1 \\
\hline
\end{tabular}

${ }^{a}$ The isotropic term $A_{l}$ taken as the bulk chemical potential was determined with the accuracy $\pm 0.1 \mathrm{eV}$ rather than $\pm 1 \mathrm{eV}$ erroneously given in paper [16] (Fig. 1).

Table 4. Values of energies of an electron. energy $\tilde{E}_{t}$.

\begin{tabular}{lcc}
\hline \multicolumn{1}{c}{ Quantity } & Tungsten W [unit] & Remarks \\
\hline \hline resonance energy $\Delta \mathrm{A}$ & $0.117 \mathrm{eV}$ & \\
maximal oscillation energy $\mathrm{E}_{\mathrm{t}}$ & 0.228 & $\mathrm{Cu}: 0.1, \mathrm{Re}: 0.2$ \\
Wigner-Seitz local energy ${ }^{\mathrm{a}}$ & -0.23 & [33] \\
unit field energy $\left(\epsilon \mathrm{F}_{\mathrm{v}}^{2} \mathrm{v}_{0} / 8 \pi\right)$ & 0.235 & \\
effective WF dipole term $\Delta \varphi$ & 0.235 & {$[16]$} \\
electron affinity $\mathrm{A}_{\mathrm{at}}$ & 0.811 & \\
electron affinity A & 0.815 & [52] \\
average WF & 4.509 & $\mathrm{Cu}: 4.62, \mathrm{Re}: 4.89$ \\
minimum WF & 4.146 & \\
local Fermi energy $\mathrm{E}_{\mathrm{F}, \mathrm{s}}$ & 4.729 & $\mathrm{Cu}: 5.78, \mathrm{Re}: 3.97$ \\
free electron energy $\mathrm{E}_{\mathrm{F}}(\tilde{\mathrm{n}})$ & 10.44 & $\mathrm{Cu}: 15.23, \mathrm{Re}: 12.47$ \\
LDA-calculated $\mathrm{E}_{\mathrm{F}}$ & 10.46 & [10] \\
unit kinetic energy $\mathrm{E}_{\mathrm{k}}\left(\mathrm{n}_{0}\right)$ & 23.60 & \\
theoretical barrier height & 23.0 & [55] \\
limiting kinetic energy $\mathrm{E}_{\mathrm{k}}\left(\mathrm{n}^{*}\right)$ & 60.18 & \\
\hline
\end{tabular}

${ }^{a}$ Energy fall at the surface Wigner-Seitz sphere with the zero of potential chosen to the surface.

$\vartheta^{-}$is an electron "coverage" ratio of $\pi \mathrm{R}_{\mathrm{a}}^{2} / \mathrm{s}_{\mathrm{h}}$ which determines the negative moment $\mathrm{M}^{-}$due to the effect of MLP internal $\triangle \mathrm{P}$ pressure. The $\vartheta^{+}$expresses the total number of broken bonds per surface atom, which determines the positive moment $\mathrm{M}^{+}$due to the surface relaxation effect. These effects correspond to the Smoluchowski electron spreading and smoothing effects.

Assuming the relation $\mathbf{D}=\varepsilon \mathbf{F}$ still valid we put arbitrarily $\varepsilon=\theta$, then the maximum virtual field
$\mathrm{F}_{\mathrm{v}}$ component in the MS dipole approximation is given by:

$$
F_{v}=\frac{4 \pi \theta e}{\varepsilon S_{0}}=\frac{4 \pi e}{S_{0}}
$$

Next, as is known from elementary electrostatics, the electrostatic potential of a neutral system of charges, as a function of point which is relatively distant from the system, should include a dipole repulsion term besides the image term of 


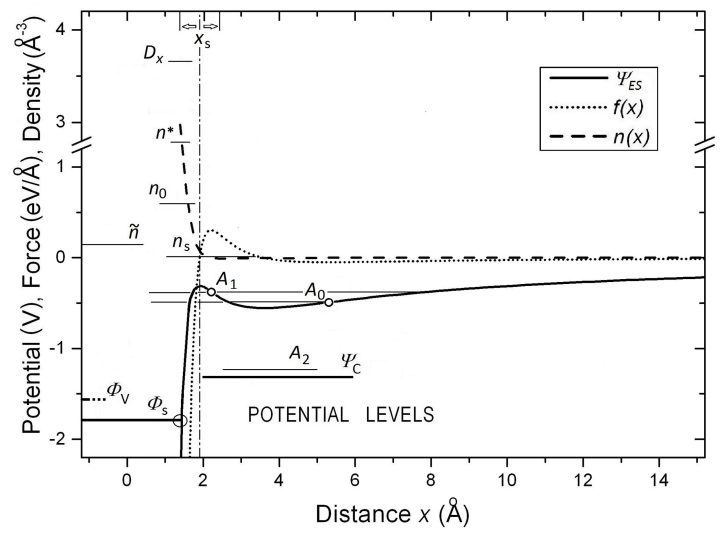

Fig. 1. "Double well" potential $\Psi_{\mathrm{ES}}$ with its first and second derivatives, and the outer and inner potential levels marked. Small circles enclose inflexion points, potential join point at $\sim 1.4 \AA$ is enclosed by larger circle. Lines A show affinity levels; chain line $\mathrm{x}_{\mathrm{S}}$ shows the position of ideal physical surface.

Coulomb attraction. Hence, any effective potential should include a dipole term $\propto \mathrm{p}_{0} \mathrm{x}^{-2}$. Then, to include the XC effects, a virtual electron e is taken as the test charge which can change its position $\mathrm{x}$ from infinity in the vacuum closer and closer to the Me/Vacuum interface, up to points just at the very physical surface. The electron must "see" a discrete surface of a few atomic layers (rather than the continuous positive jellium) for obtaining the potential energy with the XC effects included. Full expression of the potential shall include the image potential function of $-\mathrm{e} / \mathrm{x}$ completed by addition of the repulsion (dipole) potential type $\sim \mathrm{X}^{-2}$ and the image terms of repulsion/attraction of the type $\pm \alpha_{0} / \mathrm{x}^{4}$, where $\alpha_{0}$ is the dipole component of the virtual polarizability $\alpha$ of a surface atom as the electron is approaching it:

$$
\alpha=p_{0} / D_{x}=\tilde{\propto}+\alpha_{0}
$$

where $\alpha_{0}=\theta \alpha$, and $\tilde{\propto}$ is the expected MLP electron contribution to the polarizability. The image charge (unscreened) potential of the Brodie and $\mathrm{H}$ $\mathrm{D}$ approaches to the WF has the degree of homogeneity -1 of the image force approximation. This should apply to the present potential.

Finally, the effects of electron XC interactions close to the surface are evaluated in a qualitative way. The form of each XC term is based on the early interpretation [36] of the Bohr orbit precession as due to the perturbing potential $\propto \mathrm{r}^{-4}$. The field from an external electron acting on the point core of a surface atom at an $\mathrm{x}$-distance is $\mathrm{e} / \mathrm{x}^{2}$. The atomic polarization strongly affects the WF change [37], or the dipole and $\mathrm{XC}$ contributions. The polarizing action of the field makes the atom become a dipole proportional to the field strength, then the moment $\mathrm{p}_{0}$ is proportional to $\mathrm{e} \alpha_{0} / \mathrm{x}^{2}$. The potential energy of the system dipole/plasmaelectron is inversely proportional to the square of the distance electron-dipole and, simultaneously, directly proportional to the moment $\mathrm{p}_{0}$. The potential energy of the system surface-ion/outer-electron gets reduced by $\sim \alpha_{0} \mathrm{e}^{2} / \varepsilon \mathrm{x}^{4}$. Then, we may assume after $\mathrm{Li}$ and $\mathrm{Li}$ the octahedral symmetry of the valence-electrons distribution [38] between the centres of surface atoms. Taking $\varepsilon=\theta$ and $1 / 2$ as the factor of proportionality, such as the one taken in ligand-field theory for the interaction induced-dipole/ion in an octahedral system of ligands/central-ion, which is suitable for d transition metals $[25,26]$, we are driving at the electron screened potential given below.

For the surface of Me, combining the attraction and repulsion or XC terms with the dipole approximation leads to the $\mathrm{x}$-dependent expression for the MLP potential energy which tends to zero at infinity:

$$
\begin{aligned}
e \Psi_{E S}(x)= & \frac{-e^{2}}{4\left(x-x_{0}\right)}+\frac{e p_{0}}{\theta\left(x-x_{0}\right)^{2}}-\frac{e^{2} \propto_{0}}{2 \theta\left(x-x_{0}\right)^{4}} \\
& +\frac{e^{2} \propto_{0}}{2 \theta\left(x+x_{0}\right)^{4}}-\ldots
\end{aligned}
$$

where $\mathrm{x}>0, \mathrm{x} \neq \mathrm{x}_{0}$. The second term on the right-hand side of equation represents the sine-quanon dipole potential, which implies the condition $\mathrm{x} \gg 1$ for the dipole approximation. The third and the fourth terms are related to attraction and repulsion of a surface-approaching electron with a surface quasi-ion and the quasi-ion's image in the metal, respectively. This potential form takes account of XC interactions (with an inner electronimage plane assumed to be shifted by $\Delta \mathrm{x}=-\mathrm{x}_{0}$ ). Diagram in Fig. 1 shows its curve together with first 
and second derivatives. Fig. 2 shows a schema of the related surface electronic structure.

The derivative curves in Fig. 1 correspond to the force and the electronic density. Curve $-\mathrm{ed} \Psi_{\mathrm{ES}} / \mathrm{dx}$ represents the force $\mathrm{f}(\mathrm{x})$, and the outer density $\mathrm{n}$ is expressed by the $-(\theta / 4 \pi \mathrm{e}) \mathrm{d}^{2} \Psi_{\mathrm{ES}} / \mathrm{dx}^{2}$.

The electronic charge density should fulfil the Poisson equation:

$$
\frac{4 \pi n(x) e}{\varepsilon}=-\frac{d^{2}}{d x^{2}} \Psi_{E S}(x)
$$

for the infinite 2D plasma. The corresponding force also must fulfil the obvious relation:

$$
f(x)=-e \frac{d}{d x} \Psi_{E S}(x)
$$

where the negative sign results from the degree of homogeneity -1 of this potential function.

The fine structure of the potential curve including extremes and inflection points just outside the surface in the range from the ideal physical surface position $\mathrm{x}_{\mathrm{S}}$ to $\mathrm{x}_{\mathrm{c}}$ is well disclosed. The minimum of the curve is clearly connected with the metastable surface states and the electron affinity of a neutral surface atom. The maximum point of this curve coincides with the physical surface position. Omitting the two XC terms in the expression 27 results in the effective potential $\mathrm{V}_{0}\left(\mathrm{x}^{\prime}\right)$ shown in Fig. 2, which includes the dipole potential. It gives a valid approximation only for $\mathrm{x}>\mathrm{x}_{\mathrm{c}} \gg 1$, i.e. the distances, where the $\mathrm{XC}$ interactions will decay fast. Thus, the $x$-dependence of MLP electrostatic potential is expressed by:

$$
\begin{aligned}
& V(x)=\Phi_{v} \text { for } x \leqslant-d_{s} \text { in bulk phase, and: } \\
& V(x)=\Phi_{s} \text { for }-d_{s}<x<R_{d}-1 / 2 l_{h} \\
& \quad \text { in surface phase and: } \\
& V(x)=\Psi_{E S}(x) \text { for } x>R_{d}-1 / 2 l_{h}>R_{a}
\end{aligned}
$$

where the $R_{d}-1 / 2 l_{h}$ is taken as the potential join point. It is nicely seen from Fig. 1 that our potential $\Psi_{\mathrm{ES}}$ with its extremes and intersection of the inner potential $\Phi_{\mathrm{s}}$ fulfils Feynman's criterion for the existence of bound states and also has a realistic form [39]. It can be noticed that the intersection point is located just within the MS phase, with its position $1.427 \AA$ higher than the neutral atom's radius $\mathrm{R}_{\mathrm{a}}$, but not higher than $\mathrm{R}_{\mathrm{d}}$. Hence, the $\Psi_{\mathrm{ES}}(\mathrm{x})$ can be called a "double well" electrostatic potential.

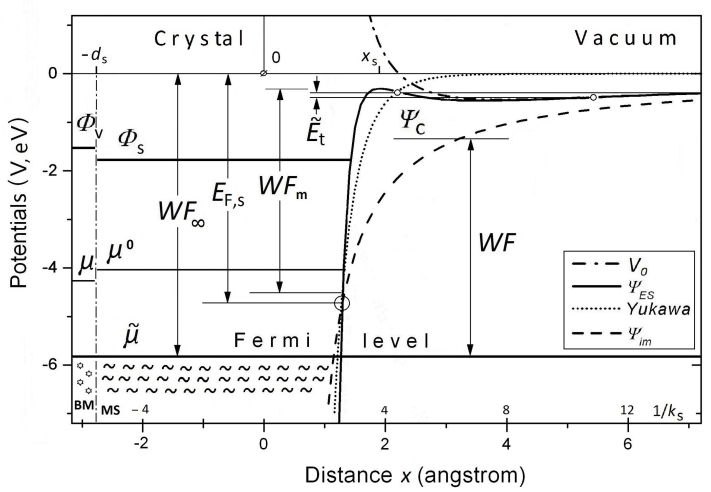

Fig. 2. Schema of electron potential relationships at metal surface. Vertical chain line shows the phase boundary plane distant by $-\mathrm{d}_{\mathrm{s}}$ from the geometrical plane. Shown are also curves of typical potentials. Larger circle shows intersection of $\Psi_{\mathrm{ES}}$ and electron-image potential $\Psi_{\text {im }}$ curves with coordinates of $1.289 \AA$ and $-4.73 \mathrm{eV}$ $\left(\approx \mathrm{E}_{\mathrm{F}, \mathrm{s}}\right)$. The intersection of Yukawa potential has coordinates $1.30 \AA$ and $-4.33 \mathrm{eV}(\approx \mu)$.

Fig. 2 shows a comparison of the screened potential $\Psi_{\mathrm{ES}}(\mathrm{x})$ with the electrochemical levels and also the typical potential forms. There are the Yukawa-type form $\left(-4 \mathrm{~A} / \mathrm{x}^{\prime}\right) \cdot \exp \left(-\mathrm{x}^{\prime} / \mathrm{x}_{0}\right) ; \Psi_{\text {im }}$ the Coulomb unscreened $-\mathrm{A} / \mathrm{x}^{\prime}$ and $\mathrm{V}_{0}$ an effective one of $-\mathrm{A} / \mathrm{x}^{\prime}+6.1 /\left(\mathrm{x}^{\prime}\right)^{2}$, where $\mathrm{A}=3.6 \mathrm{eV} \AA$. The work function which usually appears in diagrams of the literature and equals the negative of the Fermi level, is denoted $\mathrm{WF}_{\infty}$ in the figure. The $1 / \mathrm{k}_{\mathrm{s}}$ unit was computed from formula (10.34) of reference [22] putting $E_{F}(\tilde{n})=10.44 \mathrm{eV}$ and $n_{0}=\tilde{n}$.

A picture of an electron's emission which is possible owing to the presence of surface states between the $A_{0}$ and $A_{1}$ inflection points, is shown in Fig. 3. The intrinsic MS-barrier field $F$ for the reduced triangular potential $\Psi_{\Delta}$ is taken as the sum of two uniform intrinsic model fields, the image field component $-\mathrm{D}_{\mathrm{x}}$ and the maximum repulsion field $0.304 \mathrm{~V} / \AA$ at the $\mathrm{A}_{1}$ point $2.2 \AA$. The resultant field $F$ corresponds to the force $f(x)$ exerted on an outside test electron by the surface. In fact, the receptive bound states in the region between $A_{1}$ and $A_{0}$ 
are reflected by the negative density n, i.e. electron "holes".

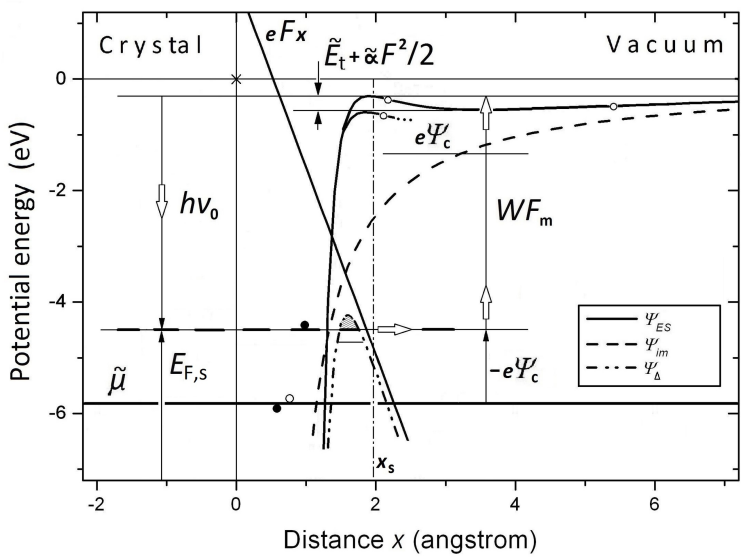

Fig. 3. Potential energy diagram simulating an MS nonstationary state during an electron's emission from metal surface. $\mathrm{F}$ is an intrinsic barrier field in the region at the physical surface distant by $\mathrm{x}_{\mathrm{s}} \pm \mathrm{x}_{0}$ (which corresponds to $\mathrm{r}_{\mathrm{s}}=3.7 \pm 1$ ). Intersection point of eFx and energy axis is at $\left(0,-\Phi_{\mathrm{s}}\right)$.

\section{Discussion}

Minimum and maximum of the potential $\Psi_{\mathrm{ES}}$ indicate the possibility of localized electron boundor surface states. MLP can behave as a self-excited resonance system and the situation is favourable to the occurrence of simple harmonic oscillation [1, 21-24]. Localized natural plasma modes are necessary, in view of Earnshaw's theorem, to stabilize the discrete charge density distribution attributed to the Me surface. Such a "dynamic" situation allows for the occurrence of simple harmonic modes in the 2D plasma medium, where the dielectric constant $\varepsilon$ must be a real number higher than zero. The tangent yz-components of the oscillation contribute to MLP longitudinal waves of conduction electrons with bulk and surface plasmons as the energy quanta which can be excited by electrons or reflected photons. Critical frequency of $\omega_{\mathrm{p}}(\mathrm{n})$ is the minimum frequency to fulfil the condition $\varepsilon \geqslant 0$. Zero of the dielectric function $\varepsilon$ is associated with the values of the $\omega_{\mathrm{p}}\left(\mathrm{n}_{\mathrm{x}}\right)$ in the selfexcited system. In quantum mechanical terms, the related $\hbar \omega_{\mathrm{p}}$ oscillation energy corresponds to a spacing between a two of discrete energy levels in the metal. The frequencies of MLP electrons are higher than $1.2 \times 10^{16} \mathrm{~s}^{-1}$ in the low density limit. For the density $\mathrm{n}_{\mathrm{s}}$ this makes $8.0 \mathrm{eV}$ which can be attributed to surface plasmons, while for the average free electron gas density $\tilde{n}$ it gives $14.54 \mathrm{eV}$ of Me bulk plasmons.

Oscillatory behaviour of measured potential profiles was observed $[40,41]$ by the STM and FIM techniques, for $\mathrm{Ag}$ nano-depositions. It was ascribed to the screened potential and Friedel oscillation around $0.12 \mathrm{eV}$, which is just about our computed value of lateral oscillation resonance-energy $\Delta \mathrm{A}$. The slope displayed a sharp decline at about 30 and the cutoff at about $15 \AA$ from step edge, respectively [41]. The normal distance $15 \AA$ is accepted as characteristic of an electron potential outside the surface [42-45]. For instance, the WF laboratory measurements give values of the work needed to remove an electron from the metal to about $15 \AA$ [45]. Above this distance from the image plane the electrostatic potential is virtually homogeneous. From the classical radial motion in the image-charge field (such as of the $\mathrm{V}_{0}(\mathrm{x})$ or the Kepler type), a distance of slightly less than $15 \AA$ can also be obtained as the limiting distance $\mathrm{x}_{\max }$ for an effective potential energy of $-2 \Delta \mathrm{A}$. In fact, the contribution of both four-order terms of the $\Psi_{\mathrm{ES}}$ diminishes very fast to tenths of a meV at $2 \mathrm{a}$, and the $\mathrm{V}_{0}(\mathrm{x})$ and $\Psi_{\mathrm{ES}}(\mathrm{x})$ curves practically converge at a distance of $5 \mathrm{a}$ (or $\geqslant 15 \AA$ ) within $\pm 0.01 \mathrm{meV}$. Such a deviation is well below any surface-atom excitation energy.

Introducing the anisotropic factor 1 via $\mathrm{p}_{0}$ to the electron screened potential $\Psi_{\mathrm{ES}}(\mathrm{x})$ makes it be the surface-structure sensitive. This permits an electron to "see" the discrete Me-surface of 2 or 3 atomic layers of the surface skin [46]. Potential $\Psi_{\mathrm{ES}}(\mathrm{x})$ is composed of an image term and three ones of the second and fourth order including the $\mathrm{XC}$ repulsion and attraction interactions. With its distinct peculiarities it fairly corresponds to the double minimum potential form as seen from Fig. 1. The maximum of $\Psi_{\mathrm{ES}}$ falls on the ideal physical surface position $\mathrm{x}_{\mathrm{s}}$. This position, on the absolute distance scale, practically coincides with the 
maximum of Tavares-Prausnitz's theoretical curve. Intersection of $\Psi_{\mathrm{ES}}$ and the inner potential $\Phi_{\mathrm{S}}$ also coincides with the primary minimum of their potential. These features of the new pseudopotential lead to a rough schema of the electronic structure of the crystal above the Fermi level and outside the MS, which is shown in Fig. 2. The electron localization and densification in the Me/Vacuum interface is accompanied by chemical bond contraction and essentially affects various physical properties of meso- and nanostructures. This is typical of under-coordinated systems [46].

The WF and affinity values obtained from the simplified formulae are in good agreement with tabular data. Other results are also in accord with quantitative data of the literature. For instance, our result for the free electron energy $E_{F}(\tilde{n})$ is in accord with the one by Paxton and van Schilfgaarde for the LDA-calculated Fermi energy of the W(110) crystal [10]. This agreement is meaningful because, as asserted by Young and Clark [47], the densest packed plane (110) with its minimum surface free energy [48] and highest WF, is the most representative of physicochemical properties for bcc metals. Putting the average density $\tilde{n}$ of free electrons and the effective dipole length 1 as the amplitude into equations 2 and 1 gives $0.228 \mathrm{eV}$ for the maximum kinetic energy $E_{t}$ of the harmonic small oscillation. This is less than the $\Delta \varphi$ term of effective WF, and both values do not exceed the maximum $0.26 \mathrm{eV}$ of the normal-energy thermionic emission energy distribution half-width [14]. Using the present data, the local electrostatic energy is obtained from the well-known formula $\epsilon \mathrm{F}^{2} \mathrm{v}_{0} / 8 \pi$, which appears to exactly be equal to the effective WF dipole term $\Delta \varphi$ and also to the unit energy at the surface Wigner-Seitz sphere [33]. It is also striking that our magnitude $4.267 \mathrm{eV}$ for the bulk chemical potential obtained by the semiempirical methods is close to the value of chemical part of the effective potential $\mathrm{V}_{\text {eff }}$ of the Lang-Kohn model in the low density limit $r_{s}=5$. This can be quantified from Fig. 3 of paper [4] by taking the difference between their electrostatic potential and the $\mathrm{V}_{\text {eff }}$, which ranges there from 4.2 to $4.4 \mathrm{eV}$ inside the positive jellium.
In conclusion, we will discuss the limiting cases in comparison to some results of widely accepted theoretical models. At the geometrical surface the spherical symmetry of frontier Wigner-Seitz cells is drastically disturbed. For metals, the electrondensity range of the Wigner-Seitz sphere parameter $r_{s}$ between 2 and 5 is proper [4-7, 48]. The MLP length parameters $d_{s}$ and L correspond to the $\mathrm{r}_{\mathrm{s}}$ range from 1 to 5.17. It should be noted after an assertion by Wojciechowski that properly describing the electronic properties of $\mathrm{d}$ transition metals requires employing two electronic density parameters [50]. Accordingly, a jellium-model density at an $r_{s}$ of 2.19 corresponds to our free electron density $\tilde{\mathrm{n}}$ which is found at $\mathrm{x} \approx 1.8 \AA$ in the $\mathrm{n}(\mathrm{x})$ curve ( $\leftrightarrow r_{s}$ of 3.4). The calculated characteristic length $\mathrm{x}_{0}$ amounts to $0.529 \AA$ which approximately equals 1 a.u. It appears equal to the result of a variational calculation by Appelbaum and Hamann that, for $r_{s}=2$, the magnitude of the origin shift $x_{0}$ tends to 1 a.u. [15]. In the low density limit, using the Gell-Mann and Brueckner approximate expression for the energy of free electron gas (formula 8.95 in $[2,7]$, a value of $-1.36 \mathrm{eV}$ is obtained for $\mathrm{r}_{\mathrm{s}}=$ $3.7\left(\leftrightarrow \mathrm{x}_{\mathrm{s}}\right)$. The number is close to our value of the outer potential energy level $\mathrm{e} \Psi_{\mathrm{c}}$ extrapolated from the intermediate point $\mathrm{x}_{\mathrm{c}}$ down to the ideal surface position $\mathrm{x}_{\mathrm{s}}$ to include the XC interactions. In the limiting case, for $x$ equal to $\mathrm{R}_{\mathrm{d}}$ higher than the atomic radius, the MLP electron density may reach a figure of $21 \times 10^{23} \mathrm{~cm}^{-3}$, which is only fifty times lower than the maximum laboratory obtainable density of inertial confinement plasma. This magnitude can be compared to the limiting figure $16 \times 10^{23} \mathrm{~cm}^{-3}$, for $r_{s}$ equal to 1 , which is obtained from the relation of Wigner-Seitz sphere radius vs electron density. (This follows Hohenberg and Kohn's assertion [7, 51]; that the relation is accurate only for $\mathrm{r}_{\mathrm{s}} \leqslant 1$ with the $n$ being determined from the expression $1 / \mathrm{n}=4 \pi \mathrm{r}_{\mathrm{s}}^{3} / 3$ ).

\section{Conclusions}

Within the thermodynamics frame, we have determined two intensive parameters of metallattice plasma, the free electron density $\tilde{n}$ and the chemical potential $\mu$. The MLP is treated as the 

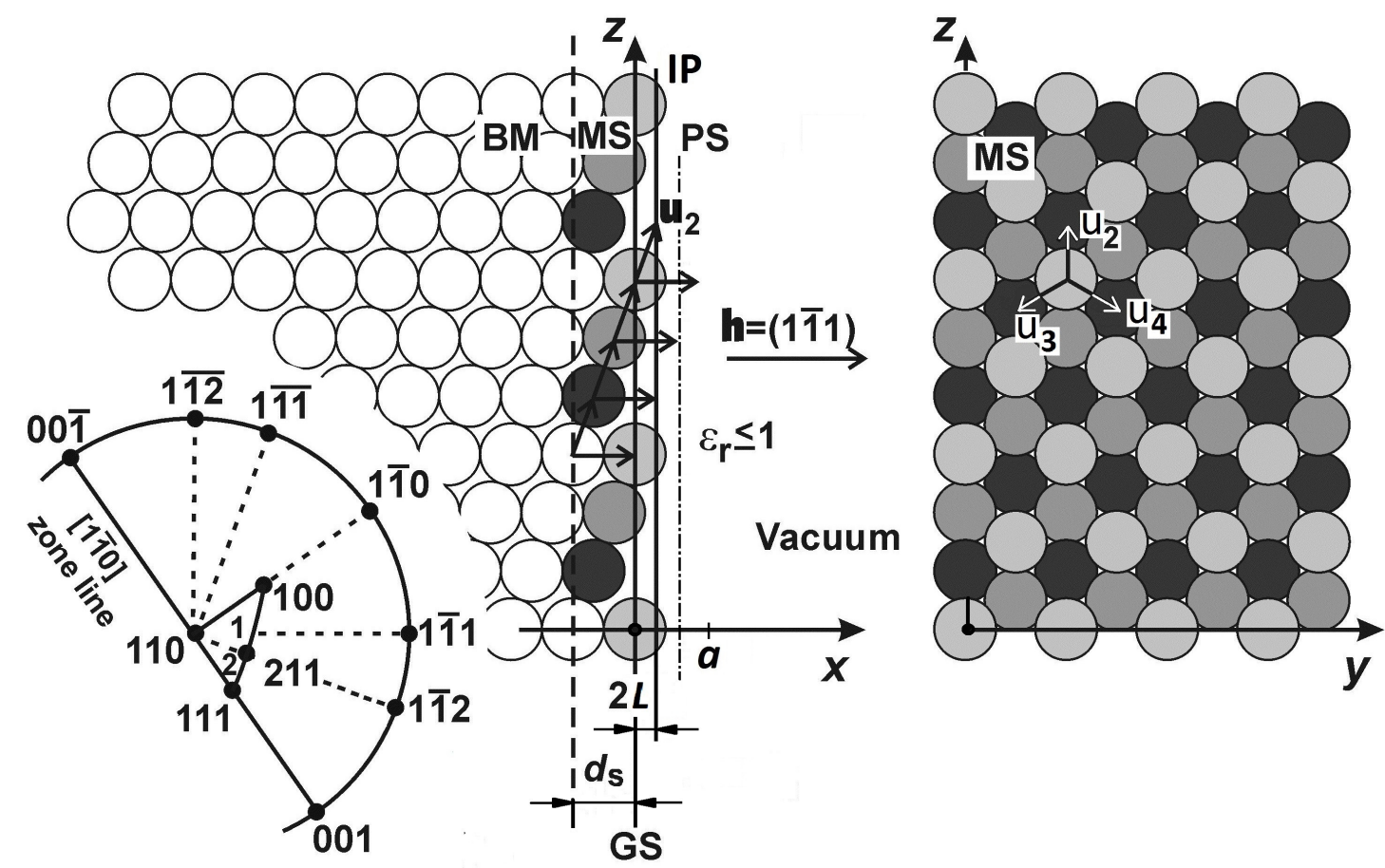

Fig. 4. Close-class plane (111) of a bcc metal with the $\mathbf{u}_{i}$ geometrical broken bonds. Left-hand part: a section layer of (110)-oriented crystal. Right-hand part: three top layers of (1111) with marked three lateral $\mathbf{u}$-bond vectors with their ends devoid of atoms. GS - the geometrical surface, IP - the primary image plane, and PS - the position of ideal physical surface.

two-component neutral system of free electrons and positive ions. We discern two phases in the metal crystal, namely the isotropic bonded 3D bulk Me phase with delocalized valence electrons and the 2D surface phase of 2 to 3 atomic layers with the dielectric constant less than unity. The phase boundary position $d_{s}$ and the plasma polarization length $\mathrm{L}$ are proposed as two limiting linear dimensions of MLP. The phase boundary was calculated in the first nearest neighbour approximation.

The novel potential $\Psi_{\mathrm{ES}}$ is exhibiting functional peculiarities; its form is close to the double minimum potential of Tavares and Prausnitz, which was calculated using theoretical methods. It is constructed as a sum of four terms of the homogeneity degree -1 . These include both the surface dipole potential and the short range interactions terms. The first and second derivatives of the potential allow for a close inspection of the forces and electron densities of the surface phase. The expected local free electron density may reach $21 \times 10^{23} \mathrm{~cm}^{-3}$, which results from the second derivative of $\Psi_{\mathrm{ES}}$.
The obtained data of the average work function and electron affinity as well as some other quantities are in good agreement with experimental and theoretical values. Calculated small-oscillation energy level spacings of 14.5 and $8.0 \mathrm{eV}$ are attributed to self-excitation of bulk Me and surface plasmons, respectively. The intrinsic surface fields range from 1 to $12 \mathrm{~V} / \AA \AA$ for the metal-lattice plasma. This MLP approach also offers a qualitative picture of the surface electronic structure and electron emission. Feasible absolute scale of the associated nanodistances from the surface is presented. This may contribute to a better understanding of theoretical models which are employed in chemical physics, catalysis and materials science of nanostructures.

\section{Acknowledgements}

We would like to thank Prof. dr hab. Ryszard Blaszczyszyn for a critical reading of a version of the manuscript and helpful comments. This research was sponsored by the University of Wroclaw under Grant No. 1010/S/IFD/14. 


\section{APPENDIX}

The phase-boundary position, or the MLP characteristic length parameter $\mathrm{d}_{\mathrm{s}}(\mathrm{hkl})$ is a function of a scalar product as defined in the first nearest neighbour approximation using the relation:

$$
d_{s}(h k l)=Q \mathbf{h} \cdot \mathbf{u}_{r} d_{h k l}
$$

where $\mathbf{u}_{\mathrm{r}}$ is the primary bond vector of the bond order relevant to bcc structure, $\mathbf{h}=(\mathrm{hkl})$ is the surface normal, and Q is the parity factor of h, k, 1 Miller indices, equal to 1 or 2 . The interplanar spacing $d_{h k l}$ is given by the crystallographic formula a/Q $\left(\mathrm{h}^{2}+\mathrm{k}^{2}+\mathrm{l}^{2}\right)^{\frac{1}{2}}$. The normal dimension $\mathrm{d}_{\mathrm{s}}$ of the MS phase is obtained taking the $\mathrm{d}_{\mathrm{hkl}}$ average value over the seven close class planes [16] of bcc structure that represent a variety of actual crystalgrain orientations. A primitive unit cell's area $S_{0}$ is also taken as the arithmetic mean of unit-cell areas of these seven planes $S_{\mathrm{hkl}}$. The $\mathrm{d}_{\mathrm{s}}$ is almost equal to the maximum value, i.e. the one obtained for the (111) plane.

The unit stereographic triangle (110)-(111)(100), at the centre of the stereographic halfprojection (110)-pole oriented, constitutes the base for bcc structure in the Mackenzie et al. theory [18]. The close-class plane of the $\{111\}$ family for a bcc Me-crystal shown in the figure is chosen to illustrate the broken ("dangling") u-bonds representation. In the first nearest neighbour approximation, the relevant 'bond order' consists of four u-bond vectors of the $<111>$ family which is relevant to the region 2 of the unit stereographic triangle. It is worth noting that, in contrast to the non-directional bonding in the homogeneous bulk Me phase, the typical surface density of the broken or unsaturated bonds is about $3.5 \times 10^{15} \mathrm{~cm}^{-2}$. Such a number and surfaces, like the one shown on the righthand side of Fig. 4 (with its metastable lattice sites) may account for the catalytic specificity of several metals.

\section{References}

[1] Ziman J.M., Principles of the Theory of Solids, Cambridge University Press, Cambridge/New York/Melbourne 1972.
[2] Harrison W.A., Pseudopotentials in The Theory of Metals, W.A. Benjamin Inc., New York/Amsterdam 1966.

[3] Schwerdtfeger P., Chem. Phys. Chem., 12 (2011), 3143.

[4] Lang N.D., Kohn W., Phys. Rev. B, 1 (1970), 4555.

[5] Lang N.D., Kohn W., Phys. Rev. B, 3 (1971), 1215.

[6] Lang N.D., Kohn W., Phys. Rev. B, 7 (1973), 3541.

[7] Hohenberg P., Kohn W., Phys. Rev. B, 136 (1964), 864.

[8] Tavares F.W., Prausnitz J.M., Colloid Polym. Sci., 282 (2004), 620.

[9] Rothschild J.A., Eizenberg M., Phys. Rev. B, 81 (2010), 224201.

[10] Brodie I., Phys. Rev. B, 51 (1995), 13660.

[11] Brodie I., Chou S.H., Yuan H., Surf. Sci., 625 (2014), 112.

[12] Halas S., Durakiewicz T., J. Phys.-Condens. Mat., 10 (1998), 10815.

[13] Durakiewicz T., Halas S., Arko A., Joyce J.J., Moore D.P., Phys. Rev. B, 64 (2001), 045101.

[14] Young R.D., Phys. Rev., 113 (1959), 110.

[15] Appelbaum J.A., Hamann D.R., Phys. Rev. B, 6 (1972), 1122.

[16] Surma S.A., Phys. Status Solidi A, 183 (2001), 307.

[17] Meyer B., The Pseudopotential Plane Wave Approach, in: Grotendorst J., Bluegel S., MARX D. (Eds.), Computational Nanoscience: Do It Yourself!, NIC Series, John von Neumann Institute for Computing, Juelich, 2006, p. 71, Fig. 3; Clark S.J., Complex structures in tetrahedrally bonded semiconductors, Ph.D. thesis, University of Edinburgh, http://cmt . dur.ac.uk/sjc/thesise-copy, 1994, p. 30, Fig. 3.4.

[18] Mackenzie J.K., Moore A.J.W., Nicholas J.F., J. Phys. Chem. Solids, 23 (1962), 185; MACKEnZIE J.K., Nicholas J.F., J. Phys. Chem. Solids, 23 (1962), 197; Nicholas J.F., An Atlas of Models of Crystal Surfaces, Gordon \& Breach, New York/London/Paris, 1965, p. 1.

[19] Smoluchowski R., Phys. Rev., 60 (1941), 661.

[20] YAVORSKI B.M., DETLAF A.A., Spravochnik po fizike, Nauka, Moscow, 1990.

[21] RAIMES S., The Wave Mechanics of Electrons in Metals, North-Holland, Amsterdam, 1961.

[22] Kittel C., Introduction to Solid State Physics, John Wiley \& Sons, 1996.

[23] Feynman R.P., Leighton R.B., Sands M., The Feynman Lectures on Physics, Addison-Wesley, Reading, Ma., 1965.

[24] Artsimovich L.A., Elementarnaya fizika plazmy, Atomizdat, Moscow, 1969.

[25] VAn MeERssche M., Feneau-Dupont J., Introduction a la cristallographie et a la chimie structural, OYEZ, Leuven/Bruxelles/Paris, 1976.

[26] Deren J., Haber J., Pampuch R., Chemia Ciala Stalego, PWN, Warszawa, 1975.

[27] Kiejna A., Wojciechowski K.F., Metal Surface Electron Physics, Pergamon, Oxford, 1996. 
[28] SWANSON L.W., DAVIS P.R., Work function measurements, in: PARK R.L., LAGALlY M.G. (Eds.), Methods of Experimental Physics, vol. 22, Solid State Physics: Surfaces, Academic Press, Orlando, Fl., 1985, p. 1.

[29] Ashcroft N.W., Mermin N.D., Solid State Physics, Holt, Rinehart and Winston, New York, 1976.

[30] VAN DER ZIEL A., Solid State Physical Electronics, Prentice Hall, Englewood Cliffs, NJ., 1976.

[31] Dobretsov L.N., Gomoyunova M.V., Emissionnaya Elektronika, Nauka, Moscow, 1966.

[32] Wigner E.P., Bardeen J., Phys. Rev., 48 (1935), 84.

[33] Bardeen J., Phys. Rev., 49 (1936), 653.

[34] Herring C., Nichols M.H., Rev. Mod. Phys., 21 (1949), 185.

[35] Koryta J., Dvorak J., Bohackova V., Lehrbuch der Elektrochemie, Springer, Wien/New York, 1975.

[36] Landau L.D., Lifshits E.M., Quantum Mechanics, Pergamon, London, 1958.

[37] Tsong T.T., Mueller E.W., Phys. Rev., 181 (1969), 530.

[38] Li W., Li D.Y., Phys. Status Solidi A, 196 (2003), 390.

[39] Feynman R.P., Statistical Mechanics. A Set of Lectures, W.A. Benjamin, New York, 1972, Fig. 2.3 and 4.1 and related text; FEYNMAN R.P., Quantum Electrodynamics, W.A. Benjamin, New York, 1961, Fig. 11.

[40] Ono M., Nishigata Y., Nishio T., Eguchi T., Hasegawa Y., Phys. Rev. Lett., 96 (2006), 016801.

[41] Ono M., Nishio T., An T., Eguchi T., Hasegawa Y., Appl. Surf. Sci. 256 (2009), 469.

[42] Durakiewicz T., Phys. Rev. B, 61 (2000), 11166.

[43] Janssens T.V.W., Castro G.R., Wandelt. K., Niemantsverdriet J.W., Phys. Rev. B, 49 (1994), 14599.
[44] KhoKonov K.B., ZAdumkin S.N., Zavisimost raboty wykhoda ot razmerov chastits, in: OvSENKO D.YE. (Ed.), Rost i Nesovershenstva Metallicheskikh Kristallov, Naukova Dumka, Kiev, 1966, p. 304.

[45] Todd C.J., Rhodin T.N., Surf. Sci., 36 (1973), 353.

[46] Sun C.Q., Prog. Solid State Ch., 35 (2007), 1.

[47] Young R.D., Clark H.E., Phys. Rev. Lett., 17 (1966), 351.

[48] Drechsler M., The Equilibrium Shape of Metal Crystals, in: VU THIEN BINH (Ed.), Surface Mobilities on Solid Materials, Plenum Press, New York/London, 1983, p. 405.

[49] EFRIMA S., Surf. Sci., 107 (1981), 337.

[50] Wojciechows Ki K.F., Mod. Phys. Lett. B, 12 (1998), 685.

[51] Gumbs G., Kogan E., Phys. Status Solidi B, 244 (2007), 3695.

[52] Mizerski W., Tablice Chemiczne, Adamantan, Warszawa, 1997.

[53] Mueller E.W., Tsong T.T., Field Ion Microscopy - Principles and Applications, American Elsevier, New York, 1969, p. 70.

[54] Kellog G.L., Tsong T.T., Cowan P., Surf. Sci., 70 (1978), 485.

[55] Smith J.R., Phys. Rev., 181 (1969), 522.
Received 2014-10-20

Accepted 2015-01-05 